\title{
The ATLAS ITk Strip Detector for the High-Luminosity LHC
}

\author{
Andrew James Blue* \\ On behalf of the ATLAS Collaboration \\ E-mail: andrew.bluedcern.ch
}

The High Luminosity Large Hadron Collider (HL-LHC) will operate at an ultimate peak instantaneous luminosity of $7.5 \times 10^{34} \mathrm{~cm}^{-2} \mathrm{~s}^{-1}$ which corresponds to approximately 200 inelastic proton-proton collisions per beam crossing (pile-up). It will be operational for more than 10 years and in that time ATLAS aims for a total data set of $4000 \mathrm{fb}^{-1}$. To operate at the higher data rates, withstand the radiation levels, and maintain low occupancy in the high pile-up environment, the current ATLAS Inner Detector (ID) will be replaced by a new Inner Tracker (ITk). The ITk will be an all-silicon tracking system that consists of a pixel detector at small radius close to the beam pipe and a large area strip tracker surrounding it. This contribution focuses on the strip region of the ITk. The central part of the strip tracker will be composed of rectangular 'short' $(2.5 \mathrm{~cm})$ and 'long' $(5 \mathrm{~cm})$ strip sensors. The forward regions of the strip tracker consist of 6 disks per side, with trapezoidal shaped microstrip sensors of various lengths and strip pitches. In response to the needs of the strip region for the ITk, highly modular structures are being studied and developed, called staves for the central region and petals for the forward regions (end-caps). These structures integrate large numbers of sensors and readout electronics, with precision light weight mechanical elements and cooling structures. A strong prototyping effort has been put in place over the course of the last years in order to optimize the ITk strip system. This contribution summarizes the R\&D activities performed by the numerous institutes within the Strips ITk collaboration showing the transition from protoyping to final production.

The 27th International Workshop on Vertex Detectors - VERTEX2018

22-26 October 2018

MGM Beach Resorts, Muttukadu, Chennai, India

\footnotetext{
*Speaker.
} 


\section{Introduction}

\section{1.1 The ATLAS Upgrade For HL-LHC}

in 2026, the High Luminosity Large Hadron Collider (HL-LHC) will operate at an ultimate peak instantaneous luminosity of $7.5 \times 10^{34} \mathrm{~cm}^{-2} \mathrm{~s}^{-1}$ which corresponds to approximately $200 \mathrm{in}$ elastic proton-proton collisions per beam crossing (pile-up) [1]. It will be operational for more than ten years and in that time ATLAS aims for a total data set of $4000 \mathrm{fb}^{-1}$.

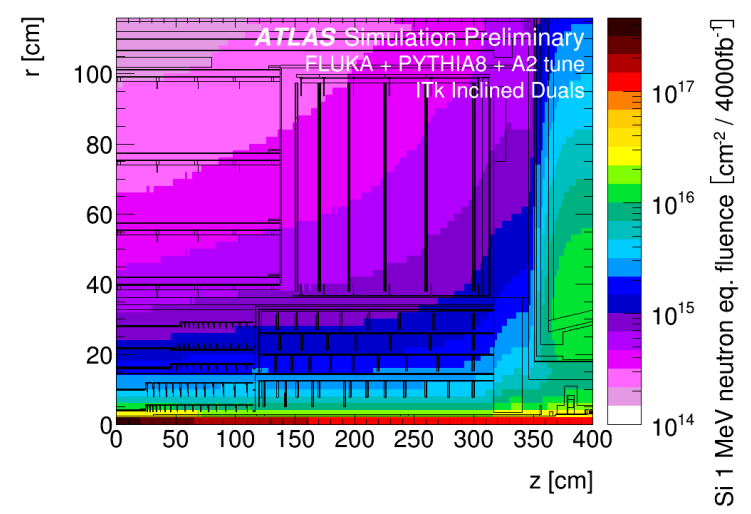

Figure 1: $1 \mathrm{MeV}$ neutron equivalent fluence per $4000 \mathrm{fb}^{-1}$ of integrated luminosity in the proposed ATLAS Inner Tracker. Here only one quadrant and only active detector elements are shown. The horizontal axis is the axis along the beam pipe with zero being the interaction point [3].

A consequence of this increased luminosity is the expected radiation damage requiring the tracking detectors to withstand a hadron fluence to over $1 \times 10^{15} 1 \mathrm{MeV}$ neutron equivalent per $\mathrm{cm}^{2}$ in the ATLAS Strip system. In addition, the current ATLAS Inner Detector (ID) [2] would not be able to cope with expected extreme data rates. Therefore to operate at the higher data rates, withstand the radiation levels (Figure 1), and maintain low occupancy in the high pile-up environment, the current ATLAS ID will be replaced by a new Inner Tracker (ITk).

\subsection{The Inner Tracker (ITk)}

The ITk will be an all-silicon tracking system that consists of a pixel detector at small radius close to the beam line and a large area strip tracker surrounding it. It will consist of five forward and central pixel layers, and four central and six forward strip layers. The ITk will approximately $6 \mathrm{~m}$ long, be around $1 \mathrm{~m}$ in radius, and cover up to a pseudo-rapidity of $\eta=4$. 


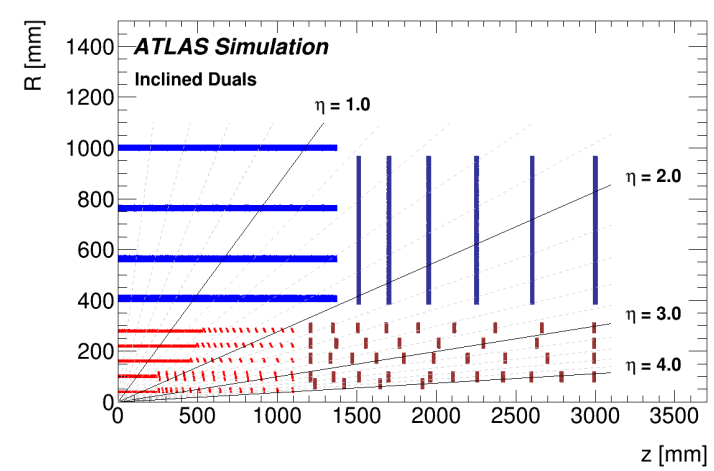

Figure 2: Schematic layout of the ITk for the HL-LHC phase of ATLAS. The active elements of the barrel and end-cap strip detector are shown in blue, for the pixel detector the sensors are shown in red for the barrel layers and in dark red for the end-cap rings [4].

Figure 2 shows a schematic of the ITk Layout, with pixel layers indicated in blue, and strip layers indicated in red. The final ITK strip system will comprise of $18 \mathrm{k}$ modules, with 60 million channels, and totalling an area of $165 \mathrm{~m}^{2}$ of Silicon.

\section{ITk Strip System}

\subsection{Sensors}

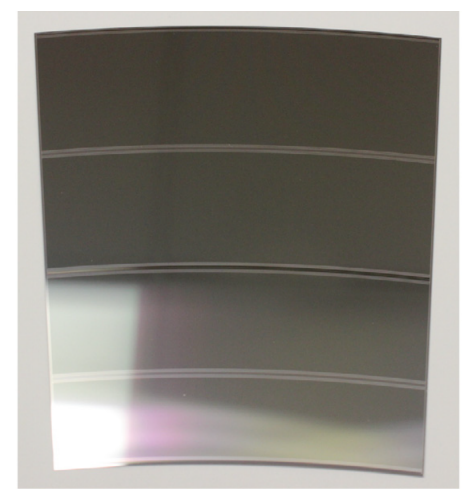

Figure 3: An R0 (innermost) sensor for the endcap (forward) region.

The Silicon sensors used by ITk strips are $320 \mu \mathrm{m}$ thick n-in-p float zone Si [5]. The use of n-in-p sensors allows for improved tolerance against radiation damage due to the lack of p-bulk type inversion. The single sided processing of the sensors also results in easier processing and handling, and therefore a reduction in cost. The central region (barrel) has only one sensor shape with $74.5 \mu \mathrm{m}$ strip pitch, and with strip lengths of $23.9 \mathrm{~mm} \& 47.8 \mathrm{~mm}$ (short \& long strips), and the forward regions (endcap) has five sensor shapes with strip lengths ranging from $8.1 \mathrm{~mm}$ to 49.9 $\mathrm{mm}$. The five sensor shapes are labeled from R0-R5, where the R0 is the innermost sensor for the endcap. An example of an R0 sensor can be seen in Figure 3. 


\subsubsection{Sensor Results}

The ITk strip community has tested several iterations of sensors, including long strip \& short strip barrel sensors and R0 endcap sensors (innermost sensor of the petal). A series of charge collection measurements from a Sr-90 source using a Alibava system [7] were made, and were found to be consistent with previous measurements over a range of irradiation sources and fluencies (Figure 4). This demonstrates that despite modifications to the design and fabrication processes of sensor types during the R\&D stage, all sensor types exhibit the charge collection criteria necessary for end of life performance.

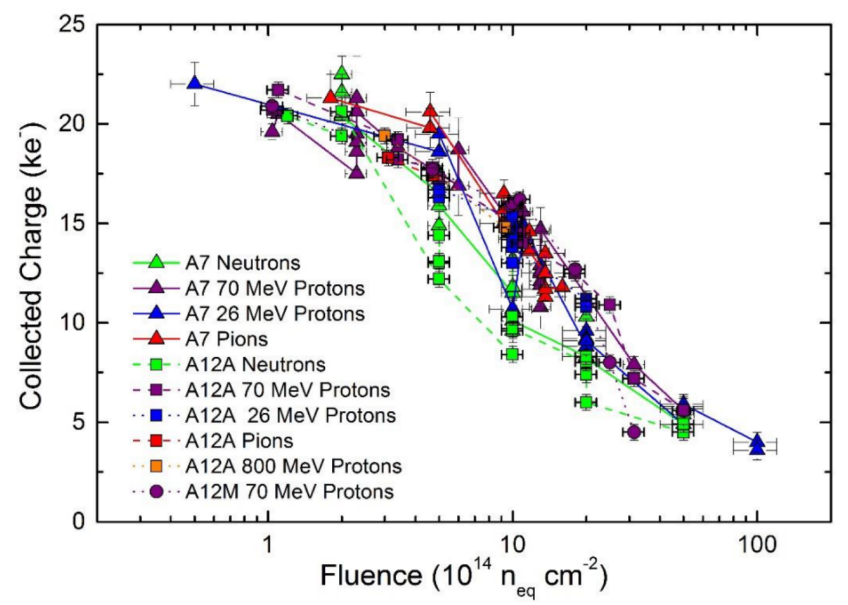

Figure 4: Collected signal charge at $500 \mathrm{~V}$ bias voltage for minimum ionising particles as a function of $1 \times 10^{15} 1 \mathrm{MeV}$ neutron equivalent per $\mathrm{cm}^{2}$ for various types of particles [6].

As well as characterising the sensors for their electrical properties, the mechanical properties of the recent R0 sensor look consistent across batches. All R0 sensors measured have a total bow (max minus min point) below $70 \mu \mathrm{m}$, which is within specification of $200 \mu \mathrm{m}$.

\subsection{Modules}

The ITk strips system is composed of modules (Figure 5) [8]. The strip modules are singlesided with hybrid circuits carrying the front-end micro-electronic ASICs glued to the sensor surface. The hybrids host the read-out electronics and power distribution board. The strip sensors are then connected to the ASIC chips via wire-bonds. Due to the variations in channel density with location in the tracker, the number of readout ASICs on a hybrid and the number of hybrids on a module varies across module types. The ABC is the front-end ASIC for the ITK strip Detector. Housed on the hybrids, they are readout by the Hybrid Controller Chip (HCC), also on the hybrid. The HCCs interface with an End of Structure Card (EoS) on the local support system the modules are attached to. 


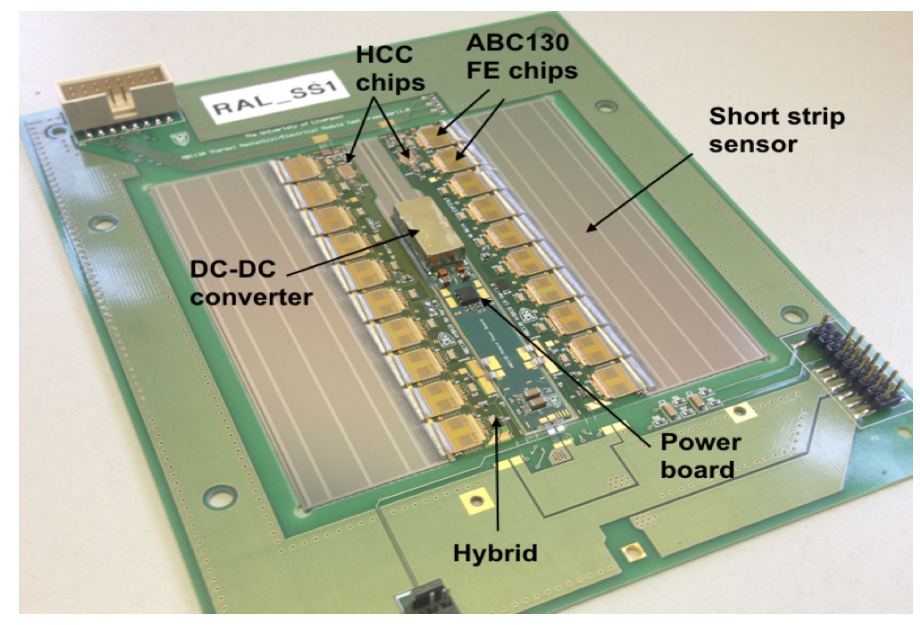

Figure 5: A fully assembled ABC130 Short-strip Barrel Module.

Modules with long strips $(47.75 \mathrm{~mm})$ make up the outer two layers of the barrel region, with modules with shorter strips $(23.9 \mathrm{~mm})$ residing in the inner two layers. In the endcap, Six module variants are used to make up the geometrical shape of a petal, with 32 petals used to make an endcap cylinder disk. Each module consists of multiple rows of strips with a pitch of $74.5 \mu \mathrm{m}$ in the central (barrel) region, and ranging from $69 \mu \mathrm{m}$ to $85 \mu \mathrm{m}$ in the forward (end-cap) regions. Despite the differing types of modules in the strips system, the main methods for assembly, readout and testing are kept consistent with each other.

\subsubsection{Module Results}

Several modules have been taken to particle test beams for characterisation. In all test beam measurements, threshold scans were performed and the tracks reconstructed with telescope data were used to determine the efficiency at each threshold. The efficiency is defined as the fraction of events in which a cluster is recorded whose centre is within $200 \mu \mathrm{m}$ from the track position as it passes the device under test. The threshold scans can be used to infer the distribution of the collected charge, as the difference between two points corresponds to the fraction of electrons producing a signal between those two threshold values. The threshold scans are then fit to a skewed error function, allowing for a determination of the most probable value (MPV) for the collected charge. The efficiency curves were evaluated at the DESY test beam for an unirradiated module with long-strip and short-strip sections at two different bias voltages, shown in Figure 6. The differences in the curves arise from the module being operated in undepleted $(250 \mathrm{~V})$ and overdepleted $(395 \mathrm{~V})$ modes, where the depletion voltage, $V_{D E P}=370 \mathrm{~V}$. The noise occupancy as a function of the threshold is fit with an error function; the shape describes the distribution well with a minimal non-Gaussian tail in the noise spectrum [6]. The signal-to-noise ratio yields values of 30 to 35 for a sensor bias voltage of $400 \mathrm{~V}$. [1] 

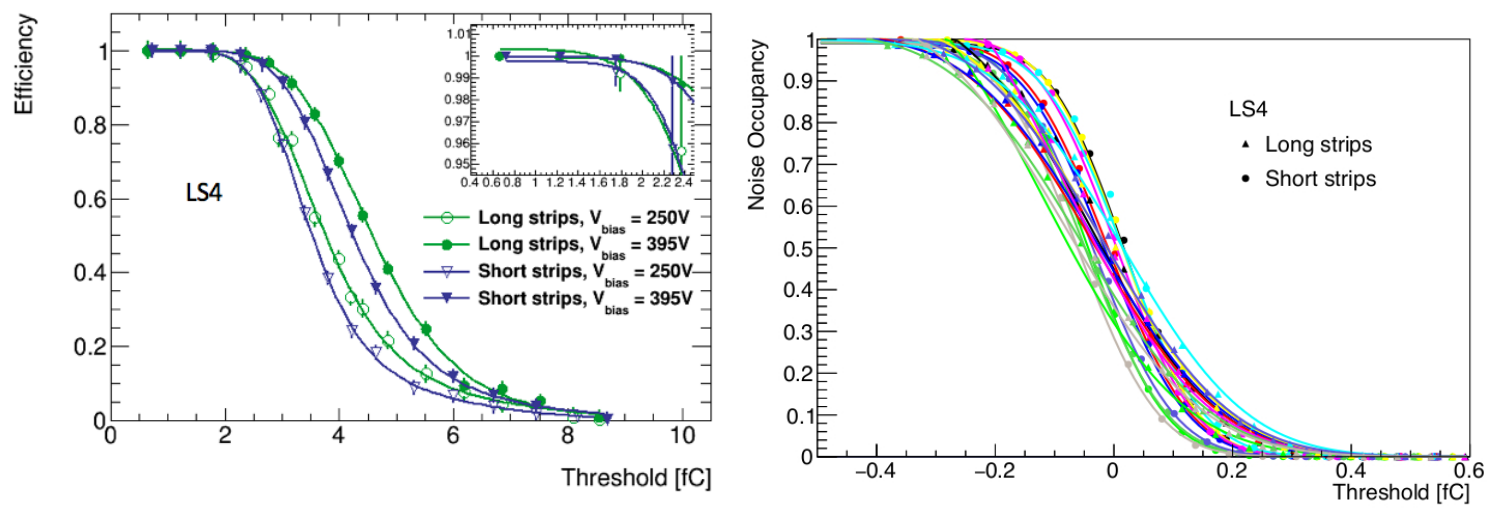

Figure 6: Left: The efficiency versus the threshold for two bias voltages and two positions on the module under test. Right: Fit of the noise occupancy vs. threshold distributions with an error function (ERFC). Each colour of the plot indicates an ASIC of the module [6].

\subsection{Local Supports}

There are 28 barrel modules on each stave (14 modules per side). Modules on each side of the stave are rotated with respect to the beam line by $26 \mathrm{mrad}$, resulting in a total rotation of $52 \mathrm{mrad}$. There are 18 endcap modules on each petal (nine modules per side). A stereo angle of $20 \mathrm{mrad}$ is directly implemented in the sensor geometry, resulting in a total stereo angle of $40 \mathrm{mrad}$. The staves and petals provide mechanical, geometric, thermal and electrical support to the modules. A schematic of a stave can be seen in Figure 7.

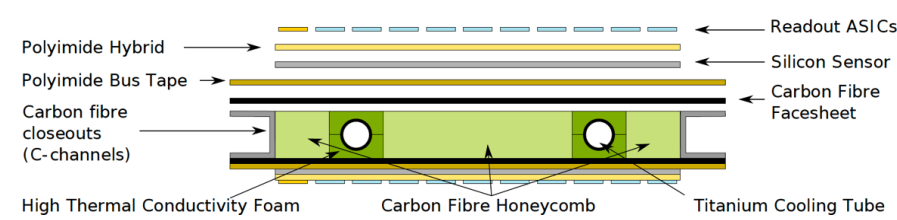

Figure 7: A schematic of the local support structure.

The local supports interface to the global support structures through a series of position locators and locking points. Modules are sandwiched on both sides of low mass carbon-fibre support structures with embedded bi-phase $\mathrm{CO}_{2}$ cooling using Titanium cooling tubes. Electrical power (both low and high voltage), TTC (Timing, Trigger and and Control) data, DCS (Detector Control System) data and measured data transfer services required by the modules are carried by a copper/kapton bus tape mounted on both sides of structure and operated by EoS (End of Substructure) card.

\subsubsection{Local Support Results}

Various prototypes have been fabricated and tested for both Stave and Petal variants. Tests include thermo-mechanical studies, stress tests and thermal cycling. 

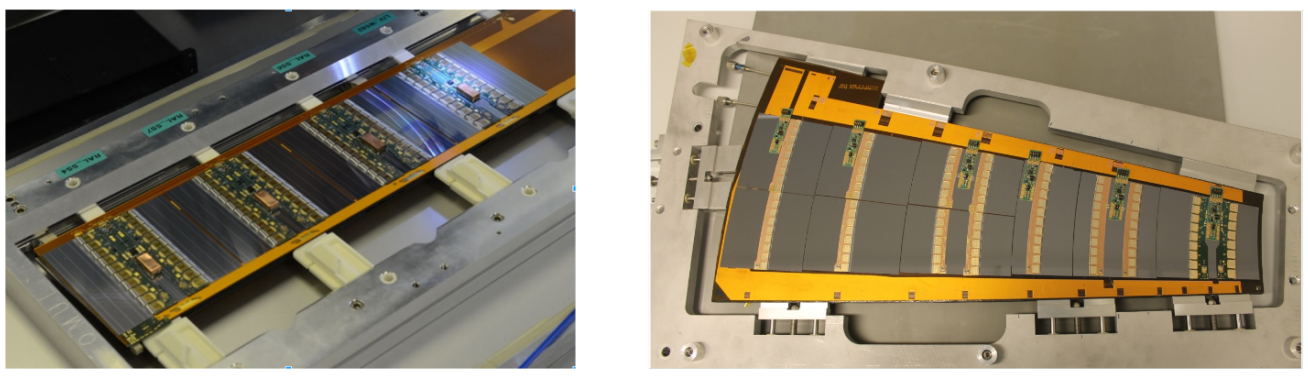

Figure 8: Photos of the thermo-mechanical (left) stave and (right) petal prototypes.

Shown in Figure 8 are examples of thermo-mechanical protypes for both stave and petal structures.

\section{Conclusions}

The High Luminosity LHC will be a challenging but exciting new stage for the LHC, and the ITk is making the transition from $R \& D$ to a pre-production phase. The ITk strip community is continuing to finalise the prototyping of components, from sensors and modules to local support and global structures. The ITk strip system is in line to begin production in 2020, with the HL-LHC program scheduled to start in 2026.

\section{References}

[1] The ATLAS Collaboration, "Technical Design Report for the ATLAS Inner Tracker Strip Detector", Technical Report CERN-LHCC-2017-005. ATLAS-TDR-025, CERN, Geneva, 04 (2017)

[2] The ATLAS Collaboration, "The ATLAS Experiment at the CERN Large Hadron Collider", JINST 3 S08003.(2008)

[3] The ATLAS Collaboration, https://twiki.cern.ch/twiki/bin/view/AtlasPublic/ RadiationSimulationPublicResults\#Phase_II_ITk_Inclined_Duals_Apri

[4] The ATLAS Collaboration, https://atlas.web.cern.ch/Atlas/GROUPS/PHYSICS/PLOTS/ITK-2018-001/

[5] B. Hommels et al., "Detailed studies of full-size ATLAS12 sensors", Nucl. Instr. Meth. Phys. Res. A831 (2016)

[6] The ATLAS Collaboration, https://atlas.web.cern.ch/Atlas/GROUPS/PHYSICS/ UPGRADE/CERN-LHCC-2017-005/

[7] Alibava Systems, 2015, Homepage http://www.alibavasystems.com

[8] S. Diez et al., "A double-sided, shield-less stave prototype for the ATLAS Upgrade strip tracker for the High Luminosity LHC", JINST 9 P02003.(2014) 